\title{
Medial plica irritation: diagnosis and treatment
}

\author{
Chad J. Griffith · Robert F. LaPrade
}

Published online: 27 November 2007

(C) Humana Press 2007

\begin{abstract}
Medial plica irritation of the knee is a very common source of anterior knee pain. Patients can complain of pain over the anteromedial aspect of their knees and describe episodes of crepitation, catching, and pseudolocking events with activities. Patients commonly have pain on physical examination upon rolling the plica fold of tissue over the anteromedial aspect of their knees and often have tight hamstrings. The majority of the patients will respond well to a non-operative treatment program consisting of quadriceps strengthening along with concurrent hamstring stretching. In cases which do not respond initially to an exercise program, an intraarticular steroid injection may be indicated. In those few patients who do not respond to a non-operative treatment program, an arthroscopic resection of their medial plica may be indicated, especially in those cases where a shelf-like plica has been found to be causing damage to the articular cartilage of the medial femoral condyle.
\end{abstract}

Keywords Plica irritation

\begin{abstract}
Anatomy
The medial plica of the knee is a thin, well-vascularized intraarticular fold of the joint lining, or synovial tissue, over the medial aspect of the knee (Fig. 1). It is present in everyone, but is more prominent in some people. It has been noted to be present as a shelf of tissue over the medial

C. J. Griffith · R. F. LaPrade ( $₫)$

Department of Orthopaedic Surgery, University of Minnesota, 2450 Riverside Avenue, R200, Minneapolis, MN 55454, USA e-mail: lapra001@umn.edu
\end{abstract}

aspect of the knee at the time of arthroscopic surgery in up to $95 \%$ of patients [6]. Proximally, it is attached to the genu articularis muscle, while distally it courses over the far medial aspect of the medial femoral condyle to attach to the distomedial aspect of the intraarticular synovial lining of the knee. At this location, it basically blends into the medial patellotibial ligament on the medial aspect of the retropatellar fat pad [9]. The medial plica is composed of relatively elastic tissues which asymptomatically conform to the changes in shape and lengths of the plica folds as the knee flexes and extends [7]. In some patients, particularly those who may have had injuries or multiple surgeries over the medial aspect of the knee, the medial synovial plica may become very thick and fibrotic and may catch over the medial aspect of the medial femoral condyle [7, 10].

In all patients, the medial synovial plica will glide over the anteromedial aspect of the medial femoral condyle with flexion and extension of the knee. In most patients, this gliding motion of the plica will occur without any symptoms, because of the high viscosity of the native synovial fluid of the knee. However, in patients with effusions, which decreases the viscosity of their synovial fluid, patients may either have crepitation or a catching of their medial synovial plica with flexion and extension of the knee. This crepitation or catching can occur with patients while going up or down stairs, squatting and bending, and other types of activities.

Since the medial synovial plica does have an attachment to the genu articularis muscle, and also an indirect attachment to the quadriceps musculature due to its attachment to the joint lining, it is dynamically controlled by the quadriceps muscles [3]. Thus, medial plica irritation is more common in patients who have poor quadriceps tone or other problems with joint muscle balance around the knee. 


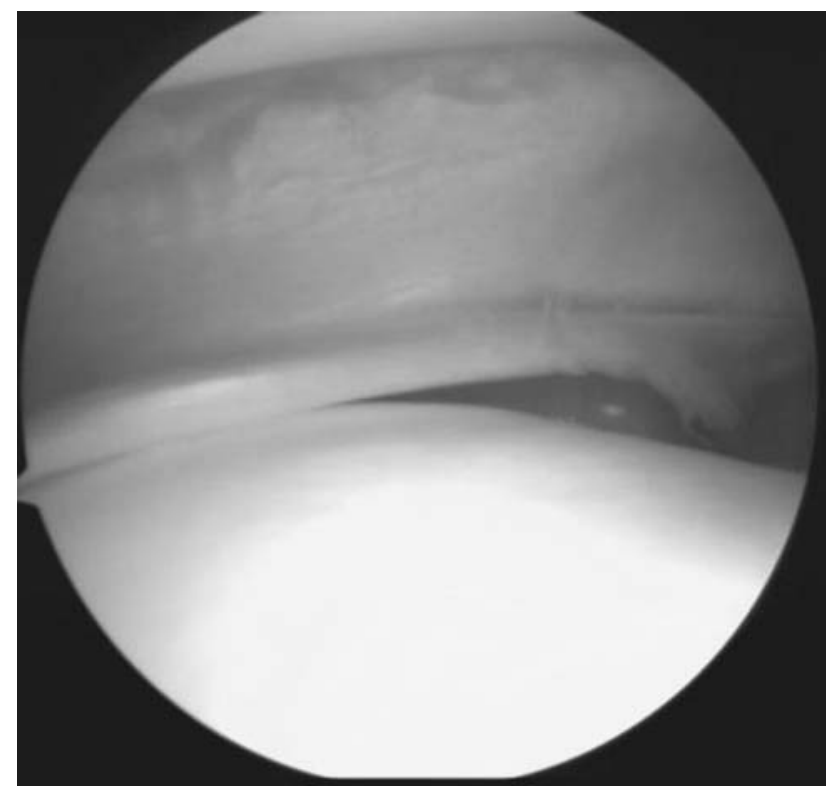

Fig. 1 Intraoperative visualization of medial synovial plica

\section{Diagnosis of medial plica pathology}

One of the most important points in diagnosing medial synovial plica pathology is obtaining an appropriate history from the patient. Patients usually describe pain which is dull, achy, and increases with activity. When asked to point to the area of their pain, they will commonly point to the proximomedial aspect of the knee, proximal to the medial joint line. While some patients may note a history of trauma to this area of the knee, most patients do not have any specific history of trauma to their medial plica. Over half of patients have a history of participating in some type of strenuous activity which requires repetitive flexion and extension motion of the knee, which then irritates their patellofemoral joint.

Most patients will complain of an achy type pain over the medial aspect of their knee, which is aggravated by activity and can be particularly bothersome at night. Their complaints of night pain over this area of the knee are due to the effects of inflammation, which can be particularly bothersome with activities. Patients most commonly complain of pain with activities which stress their patellofemoral joints, such as ascending and descending stairs, squatting and bending, and arising from a chair after sitting for an extended period of time [11]. In addition, they may note difficulty with sitting still for long periods of time without having to move and stretch their knees. They also may complain of a catch over the anteromedial aspect of their knee upon arising from a chair following prolonged periods of sitting. In some patients, plica catching may present as a pseudo-locking event to their knee when they have been sitting down for an extended period of time and they first arise. Some patients may describe these pseudolocking events as instability or catching of their patella. Clicking, giving way, and pseudo-locking have been reported in approximately $50 \%$ of all patients who present with medial plica irritation [8]. Patients who might have problems with activity-related effusions may also complain of pain over the anterior aspect of their knee. While these activity-related effusions may not be directly caused by medial plica pathology, and are more commonly due to underlying quadriceps mechanism weakness, meniscal tears, and/or osteoarthritis, but they can cause secondary medial plica irritation. In addition, patients who have had postoperative or post-injury weakness of their affected extremity may develop pain over the anteromedial aspect of their knee in the region of the medial synovial plica.

A definitive diagnosis of medial plica irritation is usually obtained by physical exam. A normal examination of the patellofemoral joint should always include an examination of the patient's medial synovial plica fold to determine if they have any irritation of this structure.

In examining the medial synovial plica, it is important to make sure that the patient is relaxed, which is usually accomplished by having the patient lie supine on the examining table with both legs relaxed. The examiner then palpates for the medial synovial plica by rolling ones fingers over the plica fold which is located between the medial border of the patella and the adductor tubercle region of the medial femoral condyle (Fig. 2). The medial synovial plica will present as a ribbon-like fold of tissue under ones finger which can be rolled directly against the underlying medial femoral condyle [12]. While some patients may have a sensation of mild pain when palpating the medial synovial plica, it is important to ascertain while performing this test if this reproduces their symptoms. It is also very important to compare the sensation to the contralateral normal knee to see if there is a difference in the amount of pain produced. It has been well demonstrated that this portion of the medial joint line and synovium is well innervated and irritation of the medial plica can be quite painful in some patients [4].

As with any other physical diagnosis, it is important to concurrently ascertain if there are other areas of pathology for structures that are located close to the medial synovial plica to confirm one's diagnosis. In acute injuries, one should make sure that there is no injury to the meniscofemoral portion of the superficial medial collateral ligament. In this instance, one would apply a valgus stress to the knee and palpate at the joint line for both any potential joint line opening to application of the valgus stress and also to see if there is any well localized pain or edema in the region of the meniscofemoral portion of the superficial medial collateral ligament (Fig. 3). In addition, in acute injuries, one should make sure that there has not been a lateral patellar 


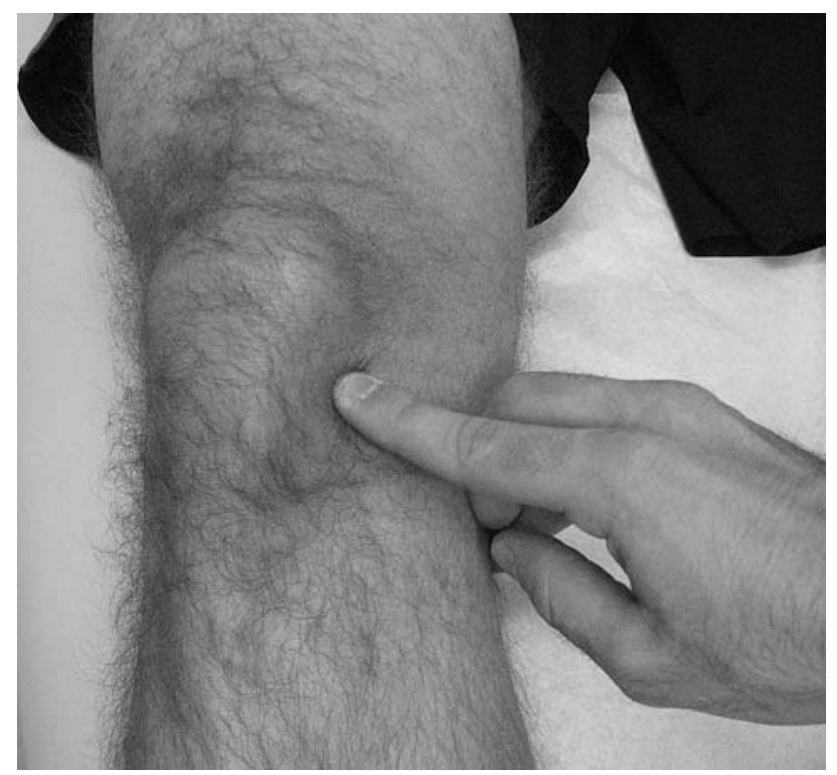

Fig. 2 Medial synovial plica palpation (Plica snap test)

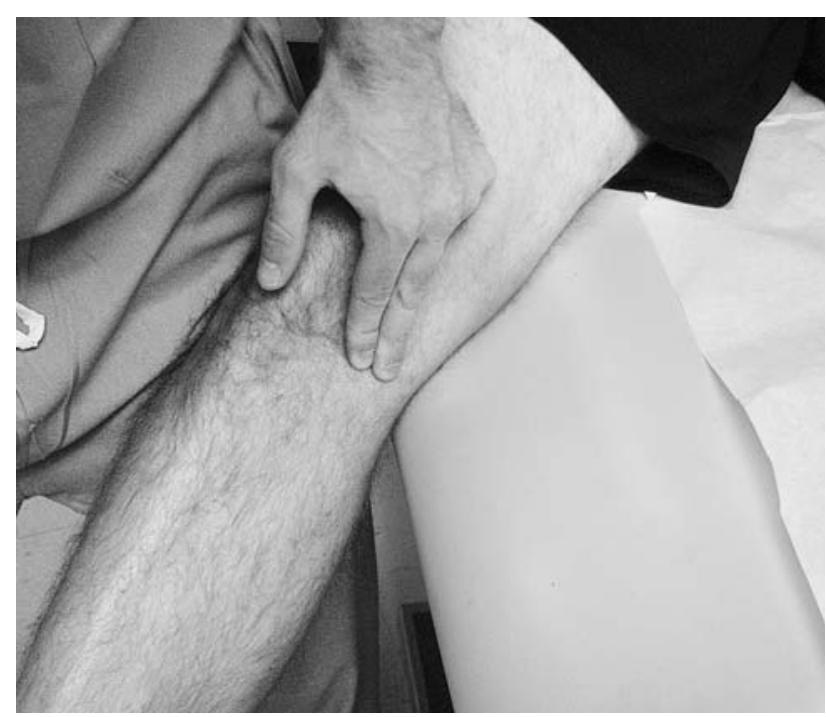

Fig. 3 Palpation of meniscofemoral portion of superficial medial collateral ligament while applying valgus stress at $30^{\circ}$ of knee flexion

subluxation episode with injury to the medial patellofemoral ligament. The lateral patellar apprehension test, performed with the knee flexed to approximately $45^{\circ}$ of knee flexion, can help to determine if there has been injury to the medial patellofemoral ligament by applying a lateral translation force to the patella when it is flexed to approximately $45^{\circ}$ of knee flexion and assessing if this translation causes pain or an apprehensive feeling like the patella will dislocate (Fig. 4). This pain should be different from pain produced when the plica is rolled under ones fingers. Further, one should make sure that the pain over the medial aspect of the knee is not directly due to localized or diffuse

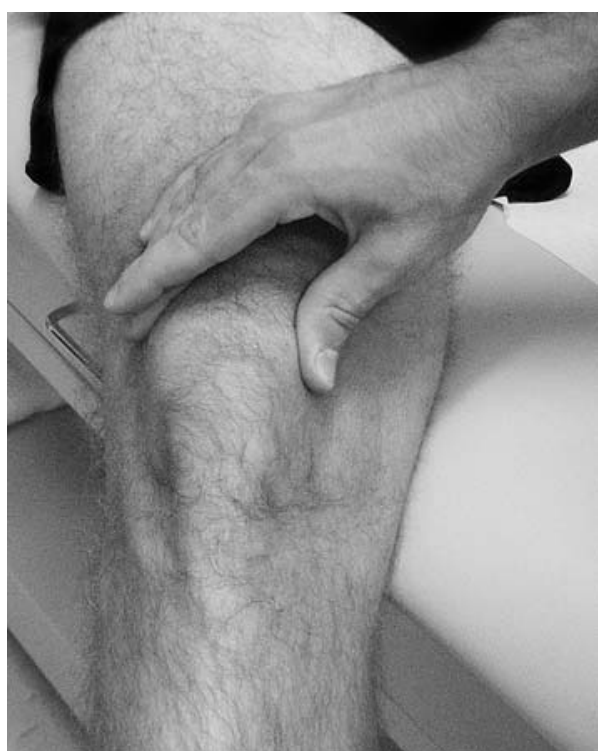

Fig. 4 Lateral translation of patella

areas of chondromalacia of the patellofemoral joint. In this instance, one would roll the superior and inferior poles of the patella both proximally and distally, as well as medially and laterally, in the trochlear groove, to determine if there is any true retropatellar crepitation with translation of the patella in the trochlear groove (Fig. 5a, b). This evaluation is different from assessing for crepitation of the patellofemoral joint with active flexion and extension of the knee (Fig. 6a, b) as many of these patients may have catching of their medial plica causing the crepitation with active flexion and extension of the knee rather than true patellofemoral chondromalacia causing this auditory occurrence. In addition, one should assess for hamstring tightness, which can cause stress to the anterior aspect of the knee, by assessing the hamstring-popliteal angle (Fig. 7) and by palpation of the main hamstring attachment sites of the knee (pes anserine bursa (Fig. 8), semimembranosus bursa (Fig. 9), and biceps bursa (Fig. 10)).

When one is not sure about the diagnosis of medial synovial plica irritation and it is difficult to determine if the patient has a true intraarticular or an extraarticular pathology over this area of the knee, one can confirm the diagnosis with an intraarticular anesthetic injection of $1 \%$ Lidocaine (Fig. 11). In this instance, the injection should be intraarticular and one should not attempt to inject directly into the medial synovial plica. This distinction is important to differentiate because the plica is actually intraarticular. If one does have good pain relief with an intraarticular anesthetic injection, one can be sure that the pathology is intraarticular rather then extraarticular over this portion of the knee. Although one series of intraplical injections reported good results [15], it has also been reported, and we also concur, that injection directly into the 
Fig. 5 Proximal and distal translation of superior and inferior poles of patella within trochlear groove. (a) Proximal translation (b) Distal translation

Fig. 6 Assessment for patellofemoral joint crepitation during active range of motion. (a) Extension (b) Flexion
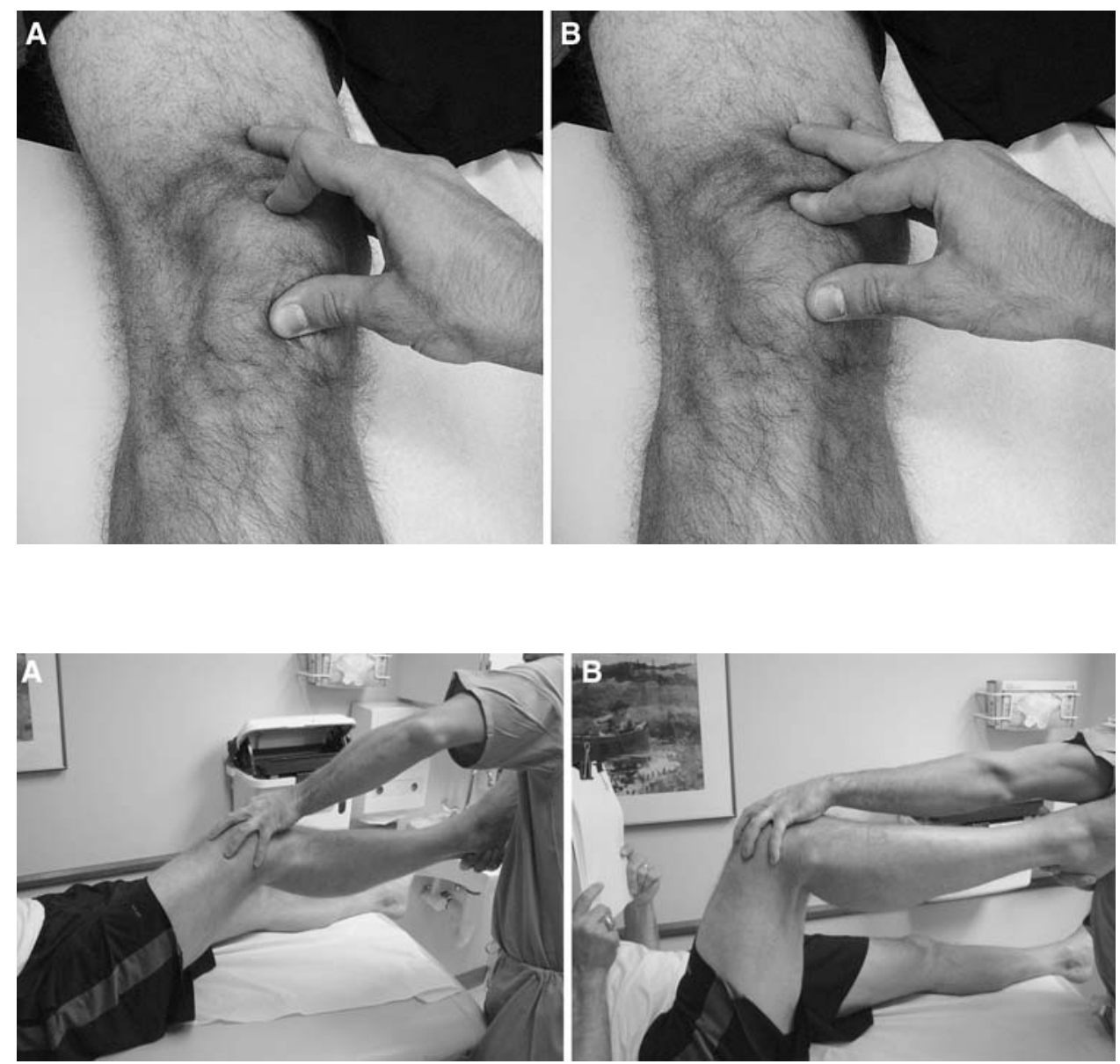

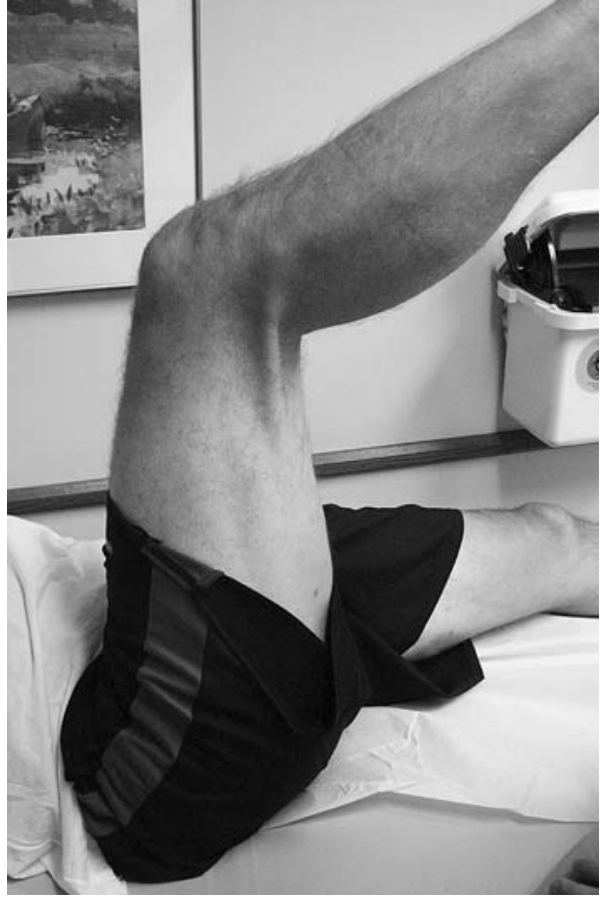

Fig. 7 Hamstring-popliteal angle

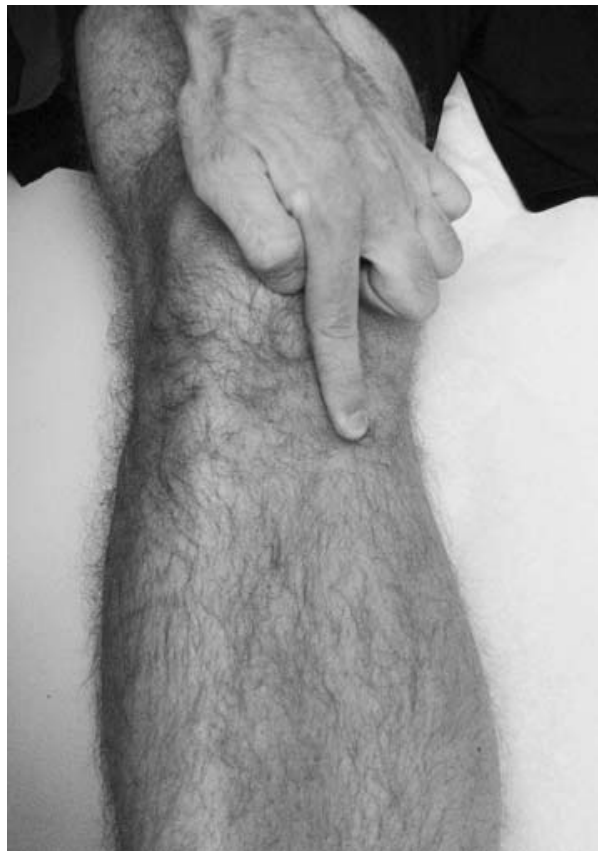

Fig. 8 Palpation of pes anserine bursae 


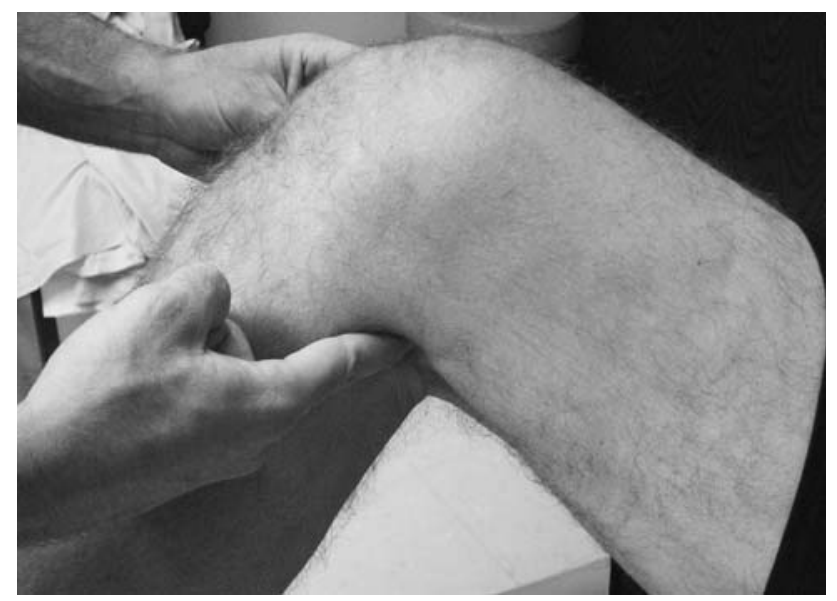

Fig. 9 Palpation of semimembranosus bursae

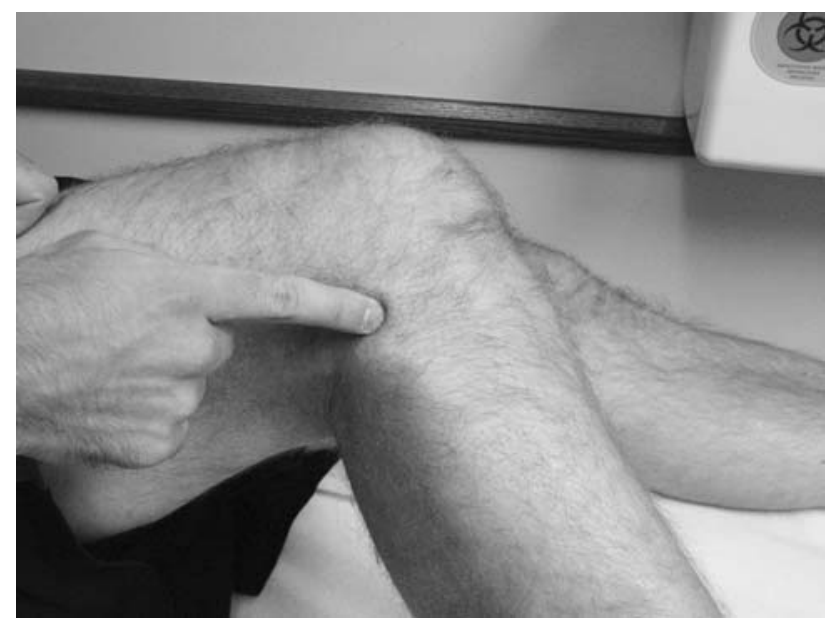

Fig. 10 Palpation of biceps femoris bursae

thin plica band is very difficult to perform and reliable placement of the needle during the injection is impossible [8]. It is generally not recommended to perform a diagnostic arthroscopy to verify that a patient has an isolated medial plica irritation, because the most successful treatments for medial plica irritation are non-operative and an arthroscopy can cause further irritation and scarring of the medial synovial plica.

It is recommended to obtain a standing AP, lateral, and $45^{\circ}$ patellar sunrise (axial) radiographs of the knee to rule out other sources of pathology. While many patients who have an irritated medial synovial plica have normal radiographs, it is important to rule out that the patients do not have any underlying arthritis, areas of osteochondritis desiccans, osteophyte formation, fractures, or other bony pathology which could be contributing to the irritation of the medial synovial plica.

In addition, diagnosis of medial plica irritation on MRI scans is non-specific [5]. The physical exam should be able

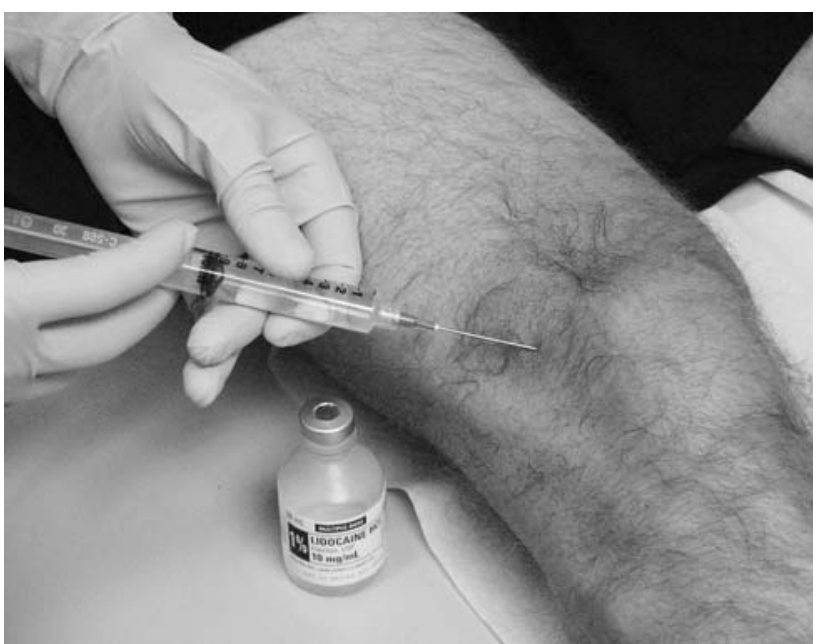

Fig. 11 Intraarticular injection of 1\% Lidocaine

to demonstrate any significant thickening and fibrosis of a medial synovial plica. MRI's are more useful in determining if there are other pathologies contributing to medial synovial plica irritation rather than in directly diagnosing pathology in this portion of the knee.

\section{Treatment of medial plica irritation}

The main treatment regimen for medial plica irritation is non-operative. For patients who have medial plica irritation as their main diagnosis without any underlying knee pathology contributing to their plica irritation, there is a very good chance that their symptoms will improve with a guided rehabilitation program $[1,12]$. The most successful rehabilitation programs focus on strengthening the quadriceps muscles, which are directly attached to the medial plica, and avoiding activities which cause medial plica irritation [3]. These exercises can include quadriceps sets (Fig. 12), straight leg raises (Fig. 13), leg presses (Fig. 14), and mini-squats, as well as, a walking program, the use of a recumbent or stationary bicycle (Fig. 15), a swimming program, or possibly an elliptical machine. Patients should work on gradually increasing strength over time to overcome any strength deficit in their quadriceps mechanism. Concurrent with this, patients should also work on a frequent hamstring stretching program throughout the day [5]. As mentioned previously, tight hamstrings can increase the force needed to extend the knee, which can be an important source of medial plica irritation. Thus, it is important to make sure that the hamstrings are stretched frequently to diminish this extra stress on the anterior part of the knee.

Most patients can utilize either a self-directed or a physical therapy guided exercise program for the first 6 8 weeks after they have been examined. In the majority of 


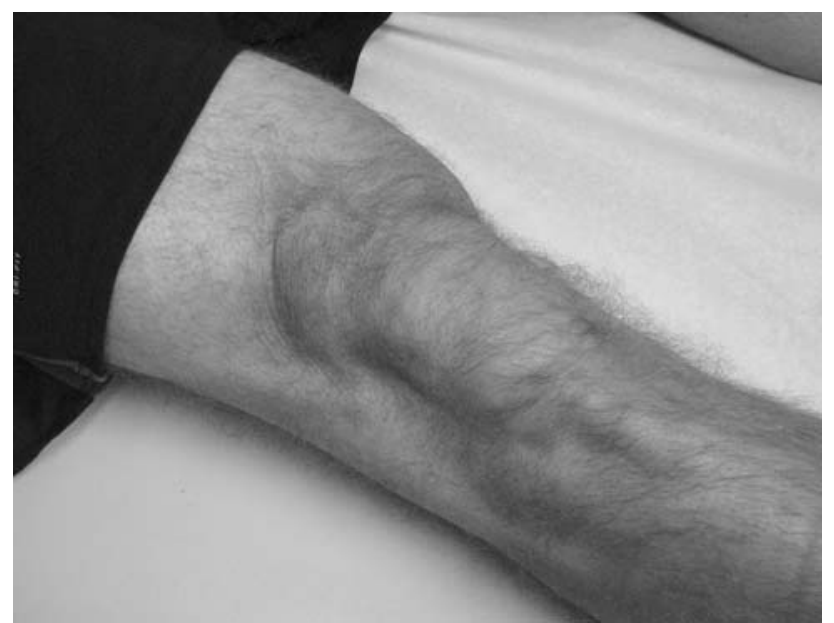

Fig. 12 Quadriceps set exercise

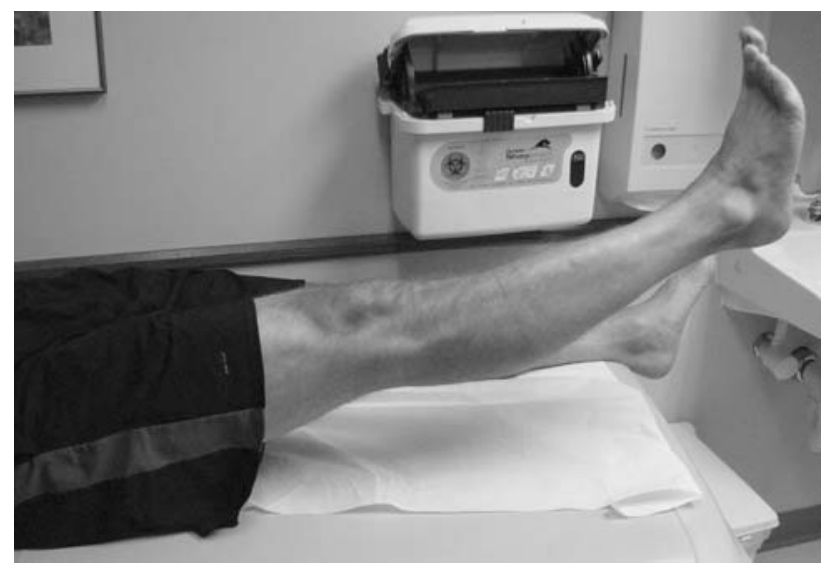

Fig. 13 Straight leg raise exercise

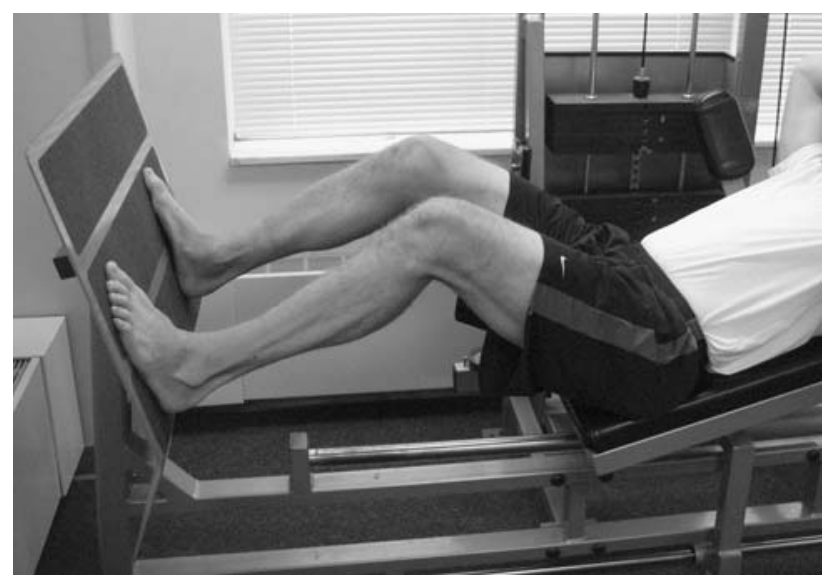

Fig. 14 Leg press exercise

circumstances, this program will alleviate the patient's symptoms and the patient can often follow-up with their physician on an as needed basis if symptoms persist after this rehabilitation program. In this therapy regimen,

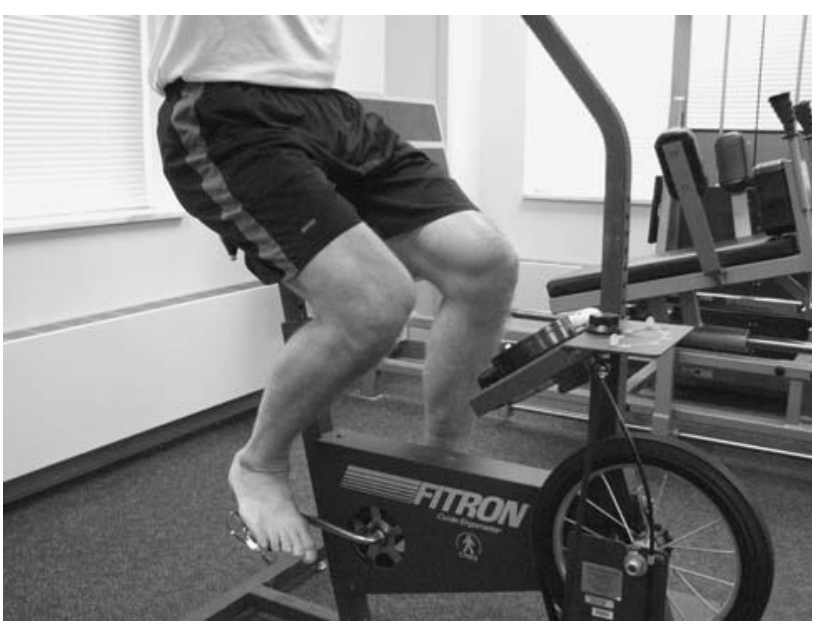

Fig. 15 Stationary bike exercise

patients participate in quadriceps strengthening exercises including a walking program, the use of a recumbent or stationary bicycle, a swimming program, or utilizing an elliptical machine. It is especially important to make sure that patients avoid knee extension exercises (Fig. 16), because open chain exercises can cause plica irritation and limit patient's improvements with an exercise regimen.

Concurrent with a good quadriceps strengthening program, it is also important to make sure that patients work on a frequent hamstring stretching program (Fig. 17). They should work on stretching their hamstrings several times a day and not just once daily. As mentioned previously, tight hamstrings place extra stress on the anterior aspect of the knee when the patient tries to extend their knee and this is a frequent cause of medial plica irritation. Thus, it is important to make sure that patients do stretch their hamstrings concurrently with strengthening their quadriceps to maximize the benefit from an exercise program.

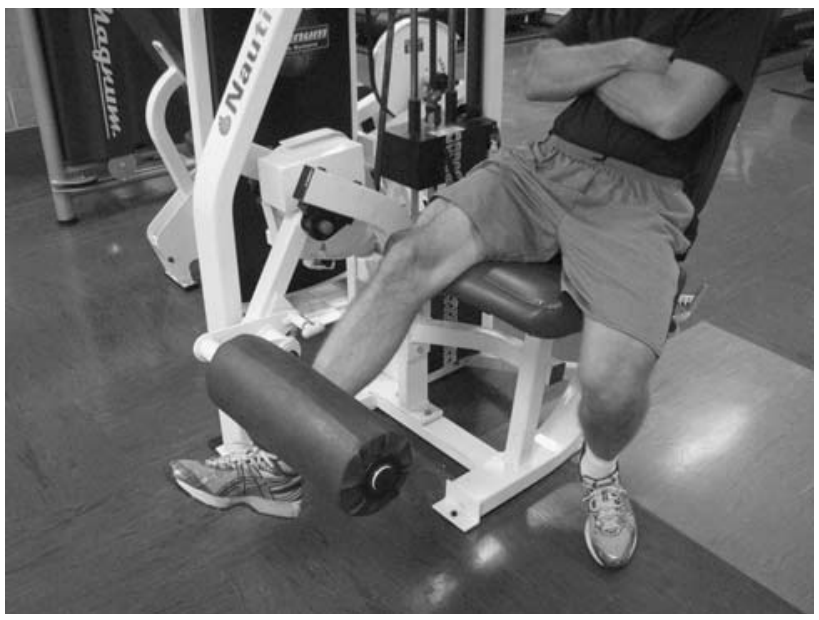

Fig. 16 Leg extension exercise 


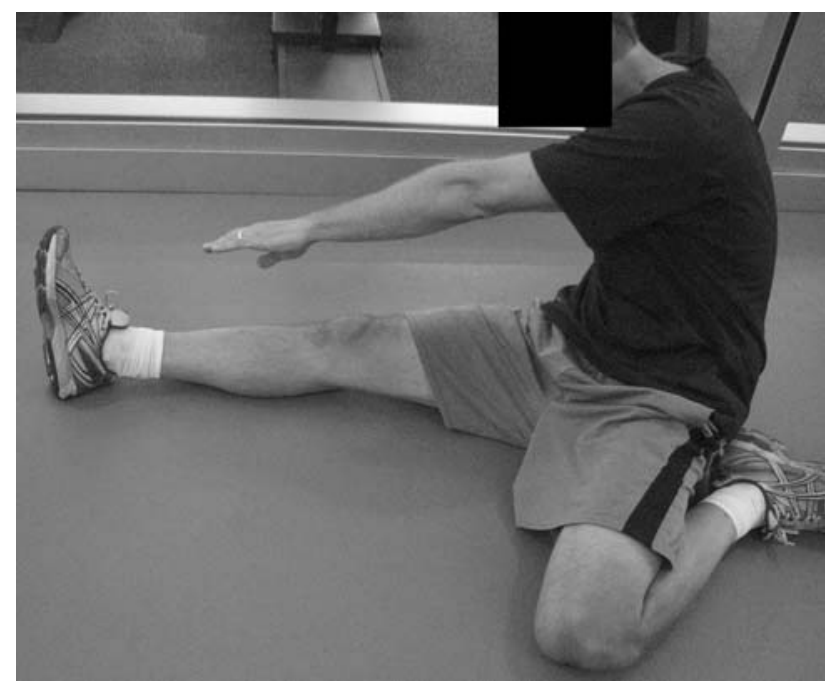

Fig. 17 Hamstring stretching

In cases where patients are not getting better with a physical therapy program, or in those patients who have such an irritated plica that a therapy program may not be beneficial directly, consideration for an intraarticular corticosteroid injection is necessary. In candidates for an intraarticular steroid injection, we perform the injection to attempt to quiet down their knee symptoms such that they can participate in an exercise program to address their medial plica irritation. It is not sufficient solely to rely on the injection to quiet down ones knee because the underlying problem of a weak quadriceps mechanism and tight hamstring muscles may persist after the injection and result in a recurrence of the medial plica irritation after the beneficial effects of the injection wear off. Thus, it is very important to make sure that the patients do participate in an exercise program, even if they have complete relief of their symptoms, after an intraarticular steroid injection to treat medial plica irritation. It is usually necessary to have the patients refrain from exercising or placing any significant stress to their knee for the first 24-48 h after their steroid injection because the knee may have more post-injection soreness. In addition, it is important to document with the patients whether or not they had good pain relief while the local anesthetic portion of the injection was working to verify that they do have an intraarticular knee cause of their knee pain.

It is rare that patients need arthroscopic surgery to treat isolated medial plica pathology, because the medial plica is a part of the joint lining and resection of it will result in the joint lining growing back. Since the body heals back this type of resection with scar, the tissue may heal back with painful scar and the patient may have more symptoms. Since the treatment of a painful plica after an arthroscopic resection can often cause patients to have more pain than they did prior to the arthroscopic resection, it is important to make sure that a patient has pathology of this area which is not responsive to an exercise program, and possibly injections, prior to consideration of resection of this tissue [2]. It is important to recognize that surgery for an irritated plica is uncommon, and historically it comprised only 2$5 \%$ of all arthroscopic surgery at a time when magnetic resonance imaging scans were not commonly used and more surgeries were performed for diagnostic reasons [5]. We have found that arthroscopic resections of irritated plicas are rarely performed today. The usual instances where one may have some benefit from resection of a medial synovial plica may be where the plica is acting as a shelf which is catching over the medial femoral condyle and causing some erosion of the articular cartilage in this area $[3,13,14]$. In these circumstances, patients may have good pain relief and decreased catching sensations in their knee after an arthroscopic resection of their medial plica. In this instance, it is usually not recommended to divide the pathologic plica and resect it totally because the pathologic plica may grow back and the patient may have recurrence of their symptoms [5]. It has also been noted that the results of arthroscopic plica excision are more successful in adolescents than in older patients [8].

Indirect treatment of the medial plica irritation may also be very beneficial to patients. In these instances, the pathology is deep within the knee. Treatment of localized areas of arthritis, meniscal tears, or other knee pathologies, may decrease the pain and swelling of the knee which resulted in secondary irritation of the medial plica. In these instances, it may be beneficial for the patient to undergo surgery to treat the secondary cause of plica irritation. In these circumstances, it is not recommended to resect the medial synovial plica, because there is a very good chance that it will get better with an exercise program after surgery and patients may not have pain relief of their plica irritation if it is resected arthroscopically.

\section{References}

1. Amatuzzi MM, Fazzi A, Varella MH. Pathologic synovial plica of the knee. Results of conservative treatment. Am J Sports Med 1990;18(5):466-9.

2. Broom MJ, Fulkerson JP. The plica syndrome: a new perspective. Orthop Clin North Am 1986;17(2):279-81.

3. Dorchak JD, Barrack RL, Kneisl JS, Alexander AH. Arthroscopic treatment of symptomatic synovial plica of the knee: long-term followup. Am J Sports Med 1991;19(5):503-7.

4. Dye SF, Vaupel GL, Dye CC. Conscious neurosensory mapping of the internal structures of the human knee without intraarticular anesthesia. Am J Sports Med 1998;26:773-7.

5. Ewing JW. Plica: pathologic or not? J Am Acad Orthop Surg 1993;1:117-21. 
6. Gurbuz H, Calpur OU, Ozcan M, Kutoglu T, Mesut R. The synovial plicae in the knee joint. Saudi Med J 2006;27(12):183942.

7. Hardaker WT, Whipple TL, Bassett FH. 3rd. Diagnosis and treatment of the plica syndrome of the knee. J Bone Joint Surg 1980;62(2):221-5.

8. Johnson DP, Eastwood DM, Witherow PJ. Symptomatic synovial plicae of the knee. J Bone Joint Surg 1993;75:1485-96.

9. Kim SJ, Choe WS. Arthroscopic findings of the synovial plicae of the knee. Arthroscopy 1997;13(1):32-41.

10. Kim SJ, Shin SJ, Koo TY. Arch type pathologic suprapatellar plica. Arthroscopy 2001;17(5):536-8.
11. LaPrade, RF. "Medial Synovial Plica Irritation", e-Medicine, http://www.emedicine.com/SPORTS/topic75.htm 2001.

12. LaPrade RF, Wentorf F. Acute knee injuries: on-the-field and sideline injuries. Phys Sportsmed 1999;27(10):55-61.

13. Lyu SR, Hsu CC. Medial plicae and degeneration of the medial femoral condyle. Arthroscopy 2006;22(1):17-26.

14. Patel D. Plica as a cause of anterior knee pain. Orthop Clin North Am 1986;17(2):273-7.

15. Rovere GD, Adair DM. Medial synovial shelf plica syndrome. Treatment by intraplical injection. Am J Sports Med 1985; 13(6):382-6. 